\title{
Interstitial microRNA miR-214 attenuates inflammation and polycystic kidney disease progression
}

\author{
Ronak Lakhia, ${ }^{1}$ Matanel Yheskel, ${ }^{1}$ Andrea Flaten, ${ }^{1}$ Harini Ramalingam, ${ }^{1}$ Karam Aboudehen, ${ }^{2}$ \\ Silvia Ferrè,, ${ }^{1,3}$ Laurence Biggers, ${ }^{1}$ Abheepsa Mishra, ${ }^{1}$ Christopher Chaney, ${ }^{1}$ Darren P. Wallace, ${ }^{4}$ \\ Thomas Carroll, ${ }^{1,5}$ Peter Igarashi, ${ }^{2}$ and Vishal Patel ${ }^{1}$ \\ 'Department of Internal Medicine, University of Texas (UT) Southwestern Medical Center, Dallas, Texas, USA. \\ ${ }^{2}$ Department of Medicine, University of Minnesota School of Medicine, Minneapolis, Minnesota, USA. ${ }^{3}$ Charles and \\ Jane Pak Center for Mineral Metabolism and Clinical Research, UT Southwestern Medical Center, Dallas, Texas, USA. \\ ${ }^{4}$ Department of Medicine and the Jared Grantham Kidney Institute, University of Kansas Medical Center, Kansas City, \\ Kansas, USA. ${ }^{5}$ Department of Molecular Biology, UT Southwestern Medical Center, Dallas, Texas, USA.
}

Renal cysts are the defining feature of autosomal dominant polycystic kidney disease (ADPKD); however, the substantial interstitial inflammation is an often-overlooked aspect of this disorder. Recent studies suggest that immune cells in the cyst microenvironment affect ADPKD progression. Here we report that microRNAs (miRNAs) are new molecular signals in this crosstalk. We found that miR-214 and its host long noncoding RNA Dnm3os are upregulated in orthologous ADPKD mouse models and cystic kidneys from humans with ADPKD. In situ hybridization revealed that interstitial cells in the cyst microenvironment are the primary source of miR-214. While genetic deletion of miR-214 does not affect kidney development or homeostasis, surprisingly, its inhibition in Pkd2- and Pkd1-mutant mice aggravates cyst growth. Mechanistically, the proinflammatory TLR4/IFN- $\gamma /$ STAT1 pathways transactivate the miR-214 host gene. miR-214, in turn as a negative feedback loop, directly inhibits TIr4. Accordingly, miR-214 deletion is associated with increased TIr4 expression and enhanced pericystic macrophage accumulation. Thus, miR-214 upregulation is a compensatory protective response in the cyst microenvironment that restrains inflammation and cyst growth.

Conflict of interest: VP has applied for patents related to the treatment of polycystic kidney disease using miR-17 inhibitors: US patent application 16/466,752 and US patent application $15 / 753,865$. VP is a member of the scientific advisory board for the PKD Foundation. VP's lab has a sponsored research agreement with Regulus Therapeutics.

Copyright: () 2020, American Society for Clinical Investigation.

Submitted: September 30, 2019

Accepted: March 11, 2020

Published: April 9, 2020.

Reference information: JCI Insight. 2020;5(7):e133785.

https://doi.org/10.1172/jici.

insight.133785.

\section{Introduction}

Autosomal dominant polycystic kidney disease (ADPKD) is among the most common monogenic disorders $(1,2)$. Mutations of PKD1 or PKD2 genes are the principal cause of ADPKD (3). Nearly $50 \%$ of individuals with ADPKD develop end-stage renal disease requiring kidney transplantation or dialysis. Despite recent significant progress, the pathogenesis of this disorder is still not fully understood, and treatment options are limited.

Large, fluid-filled, renal tubule-derived cysts are the clinical hallmark of ADPKD. Decades of research support the pivotal role of dysregulated cyst epithelial signaling in promoting cyst growth (3). However an often-overlooked aspect of ADPKD is the presence of interstitial inflammation and fibrosis. Cysts are surrounded by many types of immune cells, including M2-like macrophages and cytotoxic $\mathrm{T}\left(\mathrm{CD} 8^{+}\right)$and helper $\mathrm{T}\left(\mathrm{CD}^{+}\right)$cells, as well as cells of nonimmune origin, such as interstitial/stromal cells (4). How this altered pericystic microenvironment affects cyst progression is a question of significant interest. Several studies have reported that eliminating M2-like macrophages attenuates PKD progression in animal models (4-8). In contrast, removing $\mathrm{CD} 8^{+} \mathrm{T}$ cells from an ADPKD mouse model or excluding stroma from in vitro PKD organoid cultures aggravates cyst growth $(9,10)$. Thus, while M2-like macrophages are pathogenic, other cells in the cyst microenvironment, such as $\mathrm{CD}^{+} \mathrm{T}$ cells and stromal cells, may be protective (9). The extent and complexity of this interplay among the various cells in the niche and the underlying pathogenic or protective molecular signals are not fully known.

MicroRNAs (miRNAs) are short noncoding RNAs that bind to target mRNAs and inhibit their expression $(11,12)$. Many miRNAs are aberrantly expressed in cyst epithelium, where they mediate cyst 
epithelial dysfunction (13). For example, we have reported that the miR-17 miRNA family promotes proliferation and metabolic reprogramming of cyst epithelia (14). On the other hand, miR-21 aggravates cyst growth by suppressing cyst epithelial apoptosis (15). Others have found that miR-192/194 inhibits cyst epithelial dedifferentiation (16). Notably, our work has already resulted in the development of an anti-miR-17 drug (17). However, the full scope and impact of aberrant miRNA expression in PKD are still unknown, especially whether miRNAs regulate other aspects of PKD pathogenesis, such as the cyst microenvironment. Considering their potential therapeutic implications, the goal of this study was to identify novel miRNA modifiers of ADPKD progression.

miR-214, an evolutionarily conserved miRNA, is derived from a long noncoding RNA (lncRNA) called dynamin 3 opposite strand (DNM3OS) (18). We have previously performed global transcriptomic analysis to identify differentially expressed miRNAs and 1 cRNAs across various PKD mouse models $(14,19)$. Analysis of these data sets revealed that both miR-214 and its host lncRNA Dnm3os are upregulated in multiple PKD models. miR-214 has been linked to inflammation signaling pathways and is found in cells in the tumor microenvironment (20-23). These observations prompted us to examine the role of miR-214 in ADPKD more closely. We reasoned that miR-214 functions in the cyst microenvironment and regulates $\mathrm{PKD}$ progression.

Here, we show that miR-214 transcriptional activation is observed in both mice and humans with $\mathrm{PKD}$. The miR-214 host transcript is expressed in stromal cells in the developing kidney and in cells surrounding kidney cysts. miR-214 functions to restrain cyst-associated inflammation and the accumulation of pathogenic mannose receptor 1-positive $\left(\mathrm{MRC1}^{+}\right)$macrophages. Our work suggests that miR-214 is a protective molecular signal arising in the cyst microenvironment that attenuates cyst growth.

\section{Results}

miR-214 and its host IncRNA DNM3OS are upregulated in mouse and human PKD. miR-214 is derived from $D N M 3 O S$, an evolutionarily conserved, antisense transcript located in the 14th intron of the protein-coding gene DNM3 (Figure 1A). We have previously generated independent miRNA microarray and lncRNASeq data sets using the Ksp/Cre $P k d 1^{f / f l}(P k d 1-\mathrm{KO})$ and Pkhd1/Cre $P k d 2^{f / f l}(P k d 2-\mathrm{KO})$ orthologous genetic models of ADPKD. The Pkd1-KO model is a developmental ADPKD model in which Ksp/Cre-mediated $P k d 1$ deletion occurs in developing renal tubules beginning at around E14.5. In contrast, Pkhd1/ Cre-mediated recombination within the kidney is observed exclusively in collecting ducts. Recombination is observed in a small subset of collecting ducts at P0, but by P7 100\% of collecting ducts demonstrate Cre activity. Thus, the Pkd2-KO model can be considered an early postnatal ADPKD model (24). Analysis of these previous data sets revealed that both miR-214 and Dnm3os are upregulated in $P k d 1-\mathrm{KO}$ and $P k d 2-\mathrm{KO}$ mouse models (Figure 1A) $(14,19)$. Therefore, we decided to examine miR-214 more closely in the context of ADPKD. We began by validating the microarray and RNA-Seq data. Quantitative real-time PCR (Q-PCR) analysis showed that miR-214 and Dnm3os levels were increased by $93 \%$ and 106\%, respectively, in 35-day-old Pkd2-KO kidneys compared with age-matched control kidneys (Figure 1, B and C). Similarly, in kidneys of a slowly progressive ADPKD mouse model, which harbors a hypomorphic mutation in the $P k d 1$ gene $\left(P k d 1^{R C / R C}\right.$ ), miR-214 and Dnm3os were upregulated by $412 \%$ and $230 \%$, respectively (Figure 1 , B and C) (25). We extended these observations to human tissues and found that miR-214 and DNM3OS were increased by $127 \%$ and $135 \%$ in cystic kidney tissue from individuals with ADPKD compared with normal human kidneys (Figure 1, D and E). Thus, the upregulation of Dnm3os and miR-214 is a common feature of mouse and human PKD.

Next, we determined the location of miR-214 expression in WT and cystic kidneys. We analyzed the single-cell RNA-Seq data set generated using E18.5 mouse kidneys (26). This analysis revealed that Foxd1-derived stromal cells of nonimmune origin are the primary cell type that expresses Dnm3os in kidneys (Figure 1F). We also synthesized an RNA probe that detects miR-214 host transcript Dnm3os. In situ hybridization, using this probe, demonstrated abundant Dnm3os expression in cortical and medullary stromal cells of embryonic mouse kidneys (Figure 1G, higher magnification images are provided in Supplemental Figure 1; supplemental material available online with this article; https://doi.org/10.1172/jci.insight.133785DS1). Epithelialized renal tubules did not exhibit Dnm3os expression. Similarly, in 2-day-old postnatal kidneys, Dnm3os was also expressed in stromal cells but not in renal tubules. In kidneys from adult mice, the Dnm3os signal was less pronounced. Indeed, in agreement with these findings, a query of publicly available RNASeq data sets demonstrated robust Dnm3os expression in WT embryonic kidneys, which markedly decreased 
A
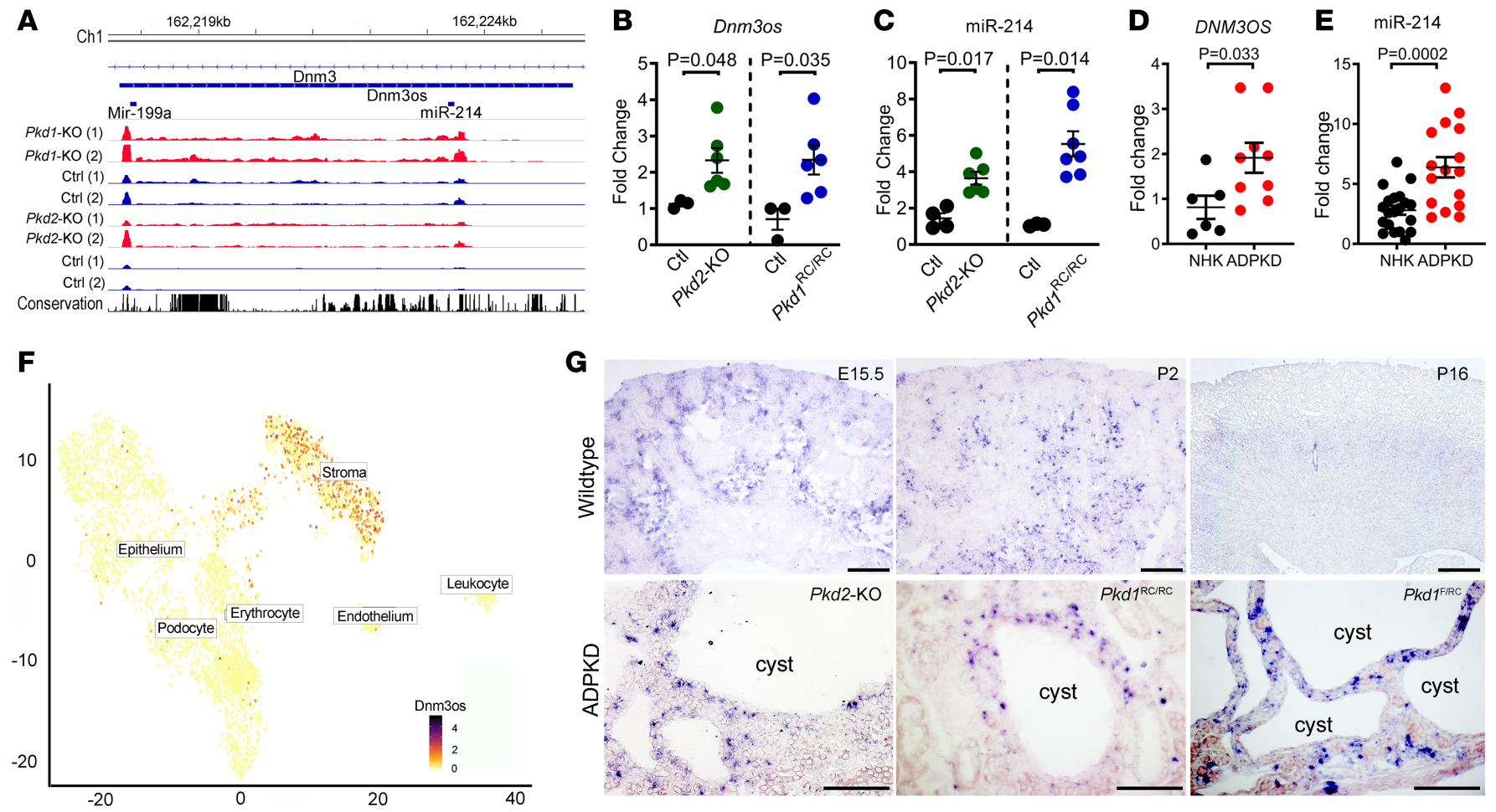

Figure 1. miR-214 and its host IncRNA DNM30S are upregulated in ADPKD. (A) IncRNA-Seq tracks showing upregulation of miR-214 and Dnm3os in 10 -dayold $P k d 1$-KO (red tracks) and 21-day-old Pkd2-KO kidneys (red tracks) compared with their respective age-matched control kidneys (blue tracks). (B and C) Q-PCR analysis showing Dnm3os and miR-214 upregulation in kidneys from 21-day-old $P k d 2-K O$ mice (green circles, $n=6$ ) and 6-month-old $P k d R^{R C / R C}$ mice (blue circles, $n=6$ ) compared with kidneys from their respective age-matched control mice (black circles $n=3-4$ ). (D and E) DNM30S and miR-214 expression was also increased in cystic kidney tissue from individuals with ADPKD compared with normal human kidney (NHK) tissue. ADPKD: $n=9$ for DNM30S and $n=16$ for miR-214. NHK: $n=6$ for DNM30S and $n=20$ for miR-214. (F) T-distributed stochastic neighbor embedding plot of single-cell RNA-Seq from E18.5 kidney shows that stromal cells are the primary cellular source of Dnm3os. (G) In situ hybridization showing Dnm3os expression in stromal cells of E15.5 and 2-day-old postnatal kidney (P2). Dnm3os signal was reduced in kidneys from 16-day-old mice (P16). Dnm3os expression in kidneys of P16 Pkhd1/Cre Pkd2f/fl (Pkd2-KO), 6-month-old $P k d 7^{R C / R C}$, and P18 Pkd $7^{f / R C}$ mice is shown at the bottom. Dnm3os expression was predominantly observed in cells surrounding cyst epithelia. Scale bars: $100 \mu \mathrm{m}$; Student's unpaired $t$ test (B, C, D, and E); error bars indicate standard error of the mean (SEM).

with maturation and was not detected in 8-week-old mouse kidneys (Supplemental Figure 2). Next, we assessed Dnm3os expression in cystic kidney tissue from 3 mouse models of ADPKD: (a) 21-day-old Pkd2KO, (b) 6-month-old $P k d 1^{R C / R C}$, and (c) 18-day-old $P k d 1^{f / R C}$ mice. Consistent with our observations in WT kidneys, we observed Dnm3os expression in cells surrounding the cysts but not in epithelial cells lining the cysts (Figure $1 \mathrm{~F}$ and Supplemental Figure 1). Thus, miR-214 host transcript is developmentally regulated, and stromal cells are the primary source of miR-214 in both WT and cystic kidneys.

miR-214 deletion aggravates cyst growth in the Pkd2-KO mouse model of ADPKD. Our observations thus far suggest an intriguing possibility that miR-214 modulates cyst growth by regulating the cyst microenvironment. To examine the role of miR-214 in ADPKD models, we first generated miR-214 ${ }^{-/-}$mice. Using homologous recombination strategy, we produced mice that harbor $l o x P$ sites flanking the miR-214 coding region within the Dnm3os gene. These mice were bred with pCMV-Cre transgenic mice to delete miR-214 in the germline (Supplemental Figure 3A). Q-PCR analysis confirmed that miR-214 was indeed absent in kidneys of miR$214^{-/-}$mice. As expected, deleting miR-214 did not affect the expression of the Dnm3os transcript upstream of miR-214 (Supplemental Figure 3B). We noted that miR-214 ${ }^{-/}$mice bred in Mendelian ratios and lived an average life span. Histological analysis of kidneys from adult miR-214 ${ }^{-/}$mice revealed normal glomerular and tubular architecture (Supplemental Figure 3C). miR-214 ${ }^{-/}$mice also displayed normal hepatic and biliary morphology (Supplemental Figure 3C). Moreover, serum creatinine, blood urea nitrogen (BUN), and serum aminotransferase levels were unchanged between miR-214 ${ }^{--}$and WT control mice (Supplemental Figure 3D). Thus, miR-214 is dispensable for mouse development as well as kidney and liver homeostasis.

We next evaluated the role of miR-214 in the context of ADPKD. We bred Pkhd1-Cre Pkd2 $2^{\mathrm{n} /+}$ mice

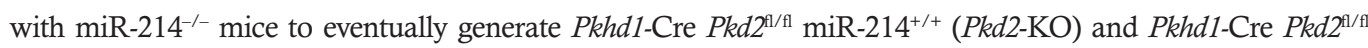


miR-214 ${ }^{-/-}\left(P k d 2-\mathrm{KO}\right.$ miR-214 $\left.{ }^{-/}\right)$mice. Q-PCR analysis confirmed that compared with WT kidneys, $P k d 2$ expression was equally reduced in both $P k d 2-\mathrm{KO}$ and $P k d 2-\mathrm{KO}$ miR-214 ${ }^{-/}$kidneys, indicating equivalent $P k d 2$ recombination (Figure 2A). On the other hand, miR-214 expression was increased in $P k d 2-K O$ kidneys compared with WT kidneys, whereas its expression was abolished in kidneys of $P k d 2-\mathrm{KO}$ miR-214 ${ }^{-/-}$mice (Figure 2B). Next, we prospectively followed a cohort of $P k d 2-\mathrm{KO}(N=22)$ and $P k d 2-\mathrm{KO}$ miR-214 ${ }^{-/-}(N=$ 21) mice to monitor disease progression. Unexpectedly, $P k d 2-\mathrm{KO}$ miR-214 ${ }^{-/-}$mice succumbed to their disease much earlier than $P k d 2-\mathrm{KO}$ mice (Figure 2C). Whereas $P k d 2-\mathrm{KO}$ mice lived for 110 days, the median survival of $P k d 2-\mathrm{KO}$ miR-214 ${ }^{-/-}$mice was reduced by $40 \%$ to only 70 days. To determine whether this reduction in survival was due to an aggravated cyst burden, we sacrificed a second cohort of 35-day-old mice. Indeed, the kidney weight/body weight ratio (KW/BW) was increased by $24 \%$, and $\mathrm{H} \& \mathrm{E}$ staining of kidney sections demonstrated more cysts in Pkd2-KO miR-214-/- kidneys compared with $P k d 2-\mathrm{KO}$ kidneys (Figure 2, D and E). BUN level was increased in $P k d 2-\mathrm{KO}$ miR-214 ${ }^{-/-}$mice compared with $P k d 2-\mathrm{KO}$ mice, but this difference did not reach statistical significance (Figure $2 \mathrm{~F}$ ). Taken together, these results suggest that miR-214 deletion aggravates $\mathrm{PKD}$ progression in $P k d 2-\mathrm{KO}$ mice.

miR-214 deletion enhances cyst-associated inflammation. To gain insights into how miR-214 inhibition promotes cyst growth, we began by comparing global transcriptome profiles of $P k d 2-\mathrm{KO}$ and $P k d 2-\mathrm{KO}$ miR-214 ${ }^{-/-}$kidneys $(n=3)$. Nine hundred seventy-two transcripts were differentially expressed between the 2 groups (Figure 3A). Unbiased pathway analysis showed that the major consequence of miR-214 deletion was the activation of tissue immune response and inflammation pathways (Figure 3B). Interstitial inflammation, in particular, the accumulation of macrophages around the cysts, is implicated in promoting cyst growth (4). Therefore, we next assessed whether miR-214 affects the quality of cyst-associated inflammation. Q-PCR analysis showed that miR-214 deletion in Pkd2-KO kidneys was associated with the upregulation of M2-like macrophage markers, arginine 1 (Arg1), monocyte chemotactic protein 1 ( Mcp1), mannose receptor $1(M r c 1)$, and chitinase-like $3(Y m 1)$ (Figure 3C). Western blot analysis also demonstrated increased MRC1 expression in Pkd2-KO miR-214 ${ }^{-/-}$kidneys compared with $P k d 2-\mathrm{KO}$ kidneys (Figure 3, $\mathrm{D}$ and $\mathrm{E})$. Moreover, immunofluorescence analysis revealed increased $\mathrm{MRC1}^{+}$macrophage accumulation around cysts in Pkd2-KO miR-214 ${ }^{-/-}$kidneys compared with $P k d 2-\mathrm{KO}$ kidneys (Figure 3F). Conversely, we found that treating RAW 264.7 cells (a macrophage cell line) with miR-214 mimics decreased the expression of M2-like macrophage markers (Supplemental Figure 4). Interestingly, miR-214 deletion did not affect the expression of markers of M1-like macrophages, T cells, or fibroblasts (Supplemental Figure 5). Thus, miR-214 deletion is associated with enhanced cyst-associated inflammatory response and augments $\mathrm{MRC}^{+}$macrophage accumulation in the cyst microenvironment.

miR-214 functions in the inflammation signaling pathways. Considering that miR-214 removal increases cyst-associated $\mathrm{MRC1}^{+}$macrophages, we reasoned that miR-214 may function to restrain inflammation signaling pathways. We began by identifying the upstream regulators of Dnm3os/miR-214 expression. We tested whether miR-214 expression is regulated by the proinflammatory (TLR4 and IFN- $\gamma / \mathrm{STAT} 1$ ) or antiinflammatory (IL-4/STAT6/3) pathways. Treating mouse embryonic fibroblast (MEF) cells with lipopolysaccharide (LPS) to activate TLR4 signaling promoted Dnm3os expression (Figure 4A). Moreover, we found that TLR4 signaling also promotes Dnm3os expression in vivo. We treated WT and Tlr $4^{\text {Lps-d }}$ mice with LPS and measured Dnm3os expression 24 hours later. Tlr $4^{\text {Lps-d }}$ have a spontaneous mutation in Tlr4 at the LPS response locus that makes them resistant to LPS. While LPS treatment increased Dnm3os expression in WT kidneys by 131\% (Figure 4B), we did not observe LPS-mediated activation of Dnm3os in kidneys of Tlr $4^{\text {Lps-d }}$ mice (Figure 4B). We found that the IFN- $\gamma /$ STAT1 pathway is upregulated in multiple mouse models of ADPKD (Supplemental Figure 6), and treating MEF or RAW 264.7 cells with IFN- $\gamma$ also induced Dnm3os expression (Figure 4C). Moreover, IFN- $\gamma$ treatment enhanced Dnm3os promoter activity in promoter luciferase assays. This stimulatory effect of IFN- $\gamma$ was lost when cells were treated with the Stat1 inhibitor fludarabine (Figure 4D). As an independent validation of our observations, analysis of ChIP-Seq data from the ENCODE database showed that treating human cancer cells (K562 cell line) with IFN- $\gamma$ results in direct Stat1 binding to the Dnm3os promoter region (Supplemental Figure 7). In contrast, IL-4 treatment failed to activate Dnm3os or miR-214 expression in MEF or RAW 264.7 cells (Supplemental Figure 8). Thus, the proinflammatory (TLR4/ IFN- $\gamma /$ STAT1), but not the antiinflammatory (IL4 $\rightarrow$ STAT6/3), signaling pathway activates miR-214 expression.

Next, we identified direct downstream targets of miR-214 in the context of PKD. We began by intersecting the RNA-Seq data ( $P k d 2-\mathrm{KO}$ vs. $P k d 2-\mathrm{KO}$ miR-214 ${ }^{-/-}$kidneys, $n=3$ each group) with a list of 
A
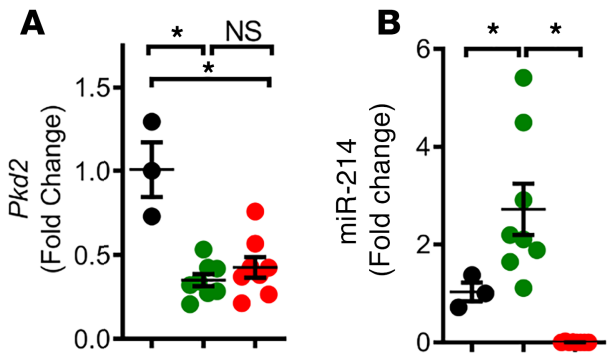

D
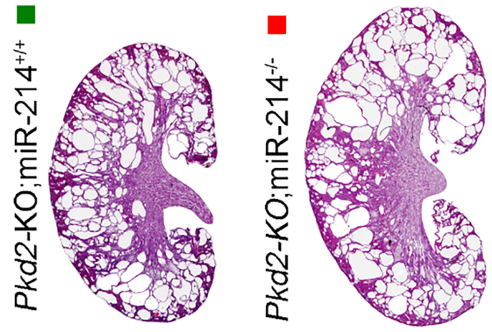

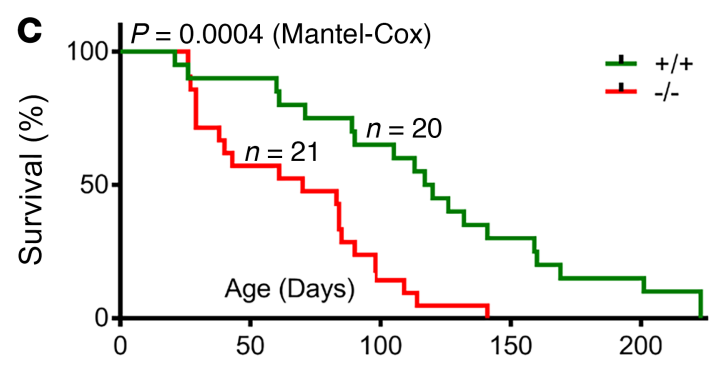

E

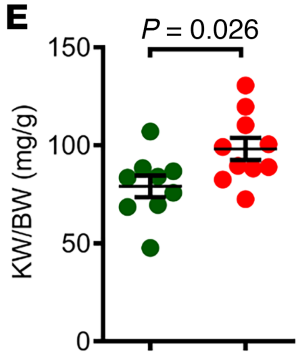

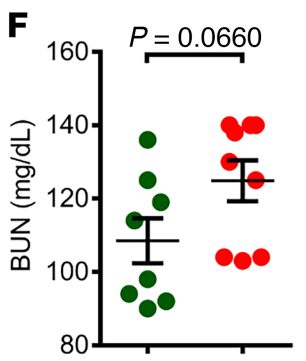

Figure 2. miR-214 deletion aggravates cyst growth in Pkd2-KO mice. To determine the role of miR-214 in ADPKD, we generated $P k d 2-K O$ miR-214 ${ }^{+/+}(P k d 2-K O)$ mice and $P k d 2-K O$ miR-214 ${ }^{/-}$mice. (A) Q-PCR analysis showing that $P k d 2$ expression was reduced equally in Pkd2-KO (green circles, $n=8$ ) and $P k d 2-K O$ miR-214 ${ }^{-1-}$ (red circles, $n=8$ ) kidneys compared with noncystic control kidneys (black circles, $n=3$ ), indicating equivalent recombination. (B) Q-PCR analysis showed miR-214 upregulation in Pkd2-KO kidneys (green circles, $n=8$ ) compared with control kidneys (black circles, $n=3$ ). As expected, miR-214 expression was abolished in Pkd2-KO miR-214 ${ }^{-1-}$ mice (red circles, $n=8$ ). (C) Kaplan-Meier survival curves of $P k d 2-K O$ miR-214-/- mice (red line, $n=21$ ) and $P k d 2-K O$ mice (green line, $n=20$ ). The median survival was reduced in $P k d 2-K O$ miR-214-/- mice compared with Pkd2-KO mice. (D) H\&Estained kidney sections of 35-day-old $P k d 2-K O$ and $P k d 2-K O$ miR-214-/- mice are shown. (E) Kidney weight/body weight (KW/BW) ratio and (F) BUN levels of 35-day-old Pkd2-KO miR-214 ${ }^{-1-}$ mice $(n=9-10)$ and $P k d 2-K O(n=8-9)$ mice are shown. ${ }^{*} P<0.05$; 1-way ANOVA, Tukey's multiple-comparisons test (A and B); Mantel-Cox test (C); Student's unpaired $t$ test (E and $\mathbf{F})$; error bars indicate SEM.

bioinformatically predicted, high-probability, direct mRNA targets of miR-214. In the process, we identified 56 mRNAs with conserved miR-214 binding sites in their 3' UTRs that were upregulated in Pkd2-KO kidneys after miR-214 deletion (Figure 5, A and B). Pathway analysis again revealed that these putative miR-214 targets were linked to inflammation-associated signaling cascades (Figure 5C). Interestingly, this list included Tlr4, raising the possibility that miR-214 functions as a negative feedback loop to restrain the TLR4 signaling pathway. Corroborating the RNA-Seq data, Q-PCR analysis showed that Tlr4 was upregulated by $38 \%$ in $P k d 2-\mathrm{KO}$ miR-214-/- kidneys compared with $P k d 2-\mathrm{KO}$ kidneys (Figure 5D). Conversely, miR-214 mimic treatment reduced Tir4 expression in mouse inner medullary collecting duct (mIMCD3), MEF, and RAW 264.7 cells (Figure 5E). To further validate Tlr4 as a direct miR-214 target, we used CRISPR/Cas9 to generate a monoclonal cell line with targeted deletion of the miR-214 binding site in Tlr4 $3^{\prime}$ UTRs ( $T r^{\Delta \text { miR-214 }}$ ) (see Methods and Supplemental Figure 9 for details). While we observed miR-214-mediated repression of Tlr4 in control cells (transfected Cas 9 but no deletion of the miR-214 binding site), this inhibition was abolished in $T l^{\Delta \text { miR-214 }}$ cell lines (Figure 5F).

In addition to TLR4, we identified 2 other miR-214 direct targets with connection to inflammation: retinoic acid-related orphan receptor (RORC) and cyclic ADP ribose hydrolase (CD38). RORC is a nuclear receptor transcription factor expressed in antiinflammatory macrophages (27). CD38 is a glycoprotein found on the surface of many immune cells. Daratumumab, which is used to treat multiple myeloma, targets CD 38 (28). We found that the expression of Rorc and $C d 38$ was increased by $74 \%$ and $59 \%$, respectively, in Pkd2-KO miR-214 ${ }^{-/}$kidneys compared with Pkd2-KO kidneys (Supplemental Figure 10). Conversely, the expression of Rorc and Cd38 was reduced by $32 \%$ and 31\% in miR-214 mimic-treated cells compared with cells treated with scramble control (Supplemental Figure 10).

miR-214 deletion aggravates inflammation and cyst growth in a slowly progressive ADPKD model. Finally, we asked whether the detrimental effects of miR-214 removal are also observed in a second slowly progressive ADPKD mouse model. To address this question, we deleted miR-214 in $P k d 1^{R C / R C}$ mice (25). 
A
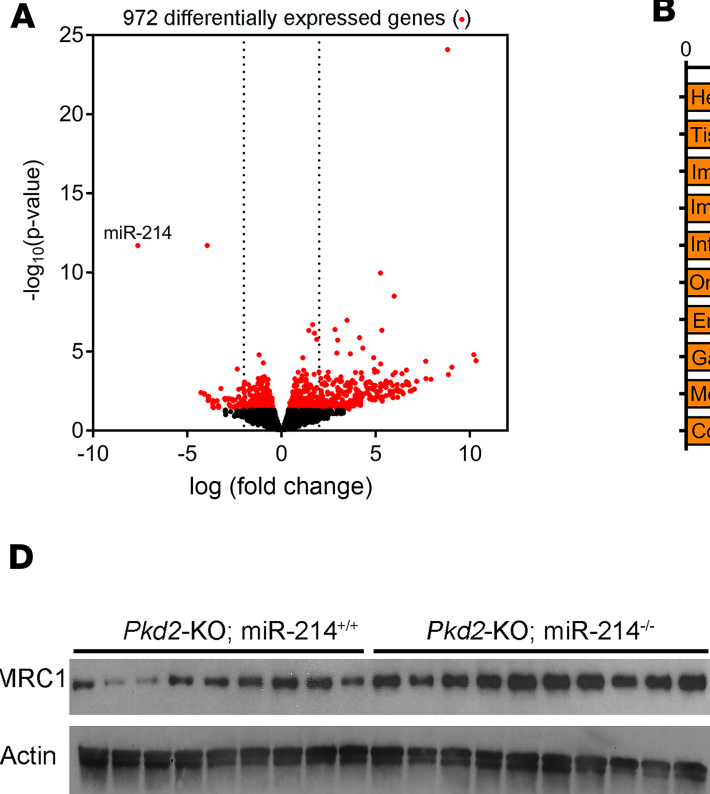

B

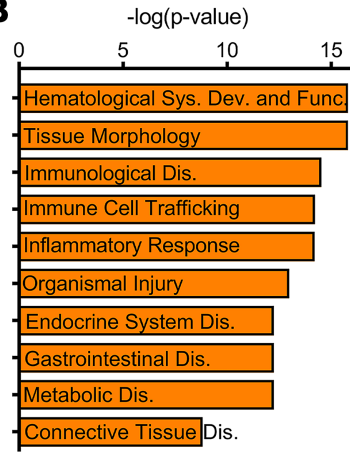

C

Pkd2-KO; miR-214 ${ }^{+/+}$

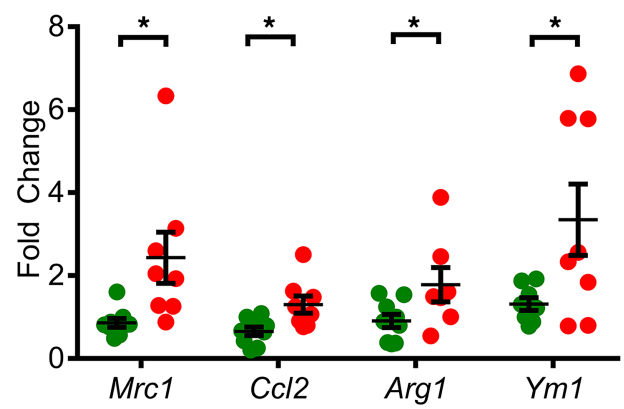

E

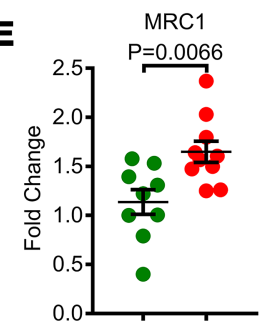

$\mathbf{F}$

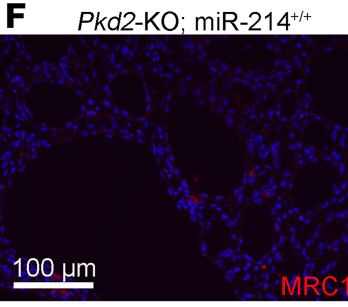

Pkd2-KO; miR-214

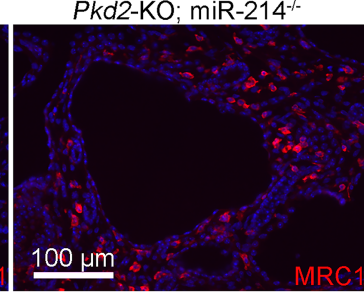

Figure 3. miR-214 deletion augments cyst-associated inflammation. (A) Volcano plot showing 972 differentially expressed genes between $P k d 2-K O$ miR-214 ${ }^{--}$and $P k d 2-K O$ kidneys ( $n=3 /$ group). (B) Unbiased pathway analysis revealed that inflammation-associated pathways are among the top dysregulated pathways in Pkd2-KO miR-214 ${ }^{--}$kidneys compared with Pkd2-KO kidneys. (C) Q-PCR analysis showed that the expression of M2-like macrophage markers Mrc1, Ccl2, Arg1, and Ym1 is increased in Pkd2-KO miR-214 ${ }^{-/-}$kidneys $(n=8)$ compared with Pkd2-KO kidneys $(n=8)$. Western blot (D) and quantification (E) demonstrated increased MRC1 expression in Pkd2-KO miR-214 ${ }^{--}$kidneys $(n=9)$ compared with Pkd2-KO kidneys $(n=9)$. (F) Immunofluorescence staining revealed enhanced accumulation of MRC1+ macrophages (red) surrounding cyst epithelial cells in $P k d 2-K O$ miR-214 ${ }^{-1-}$ kidneys $(n=5)$. The sections are costained with DAPI (blue). ${ }^{*} P<0.05$; Student's unpaired $t$ test (C and $\left.\mathbf{E}\right)$; error bars indicate SEM.

We crossed $P k d 1^{R C / R C}$ mice with miR-214 ${ }^{-/-}$mice to generate $P k d 1^{R C / R C}$ mice in which miR-214 is present $\left(P k d 1^{R C / R C}\right)$ and mice with deletion of either one or both alleles of miR-214 ( $P k d 1^{R C / R C}$ miR-214 $\left.{ }^{\mathrm{mut}}\right)$. The $P k d 1^{R C / R C}$ miR-214 ${ }^{\mathrm{mut}}$ group consisted of $4 P k d 1^{R C / R C}$ miR-214 ${ }^{-/-}$mice and $8 P k d 1^{R C / R C}$ miR-214 ${ }^{+/-}$mice. Unexpectedly, $2 P k d 1^{R C / R C}$ miR-214 ${ }^{-/-}$mice spontaneously died before 4 months of age, precluding the analysis of their cystic phenotype. Q-PCR analysis showed that miR-214 expression was increased in $P k d 1^{R C / R C}$ mice kidneys, but it was reduced in $P k d 1^{R C / R C}$ miR-214 ${ }^{\text {mut }}$ kidneys (Figure 6A). We assessed the cystic phenotype of a cohort of $P k d 1^{R C / R C}$ and $P k d 1^{R C / R C}$ miR-214 $4^{\text {mut }}$ mice by performing kidney MRIs at 4 months of age. Compared with $P k d 1^{R C / R C}$ mice, MRI-assessed total kidney volume (adjusted to body weight) was increased by $21 \%$ in 4 -month-old $P k d 1^{R C / R C}$ miR- $214^{\text {mut }}$ mice (Figure $6, \mathrm{~B}$ and C). There was no difference in phenotype between mice with deletion of either one or both alleles of miR-214. These mice were sacrificed and kidneys were obtained for molecular analysis. The expression of miR-214 targets Tlr4 (up by 323\%), Rorc (up by 183\%), Cd38 (up by 364\%), and Cd84 (up by 342\%) was increased in $P k d 1^{R C / R C}$ miR-214 ${ }^{\text {mut }}$ kidneys compared with $P k d 1^{R C / R C}$ kidneys (Figure 6D). Thus, similar to our observations in $P k d 2-\mathrm{KO}$ mice, miR-214 deletion increased inflammation and cyst burden in $P k d 1^{R C / R C}$ mice.

\section{Discussion}

The defining characteristic of ADPKD is the large, fluid-filled, kidney tubule-derived cysts. Abnormal cellular signaling in renal tubules is undoubtedly the primary driving force for cyst formation. However, several recent studies suggest that cells surrounding the cysts create a microenvironment that also affects tubular cyst growth (5). The cyst microenvironment - perhaps analogous to cancer microenvironment is a heterogeneous cell population of immune and nonimmune origin. Initial studies have suggested a complex niche where some cells promote (e.g., M2-like macrophages) while others abate (e.g., $\mathrm{CD}^{+} \mathrm{T}$ cells) cyst growth. Our work adds to this complexity and implicates miR-214 and, by extension, stromal cells as new protective players in the cyst microenvironment.

We have provided several new insights linking miR-214 to ADPKD progression. First, miR-214 is robustly and consistently upregulated both in murine and human forms of ADPKD. miR-214 is derived from the 
A

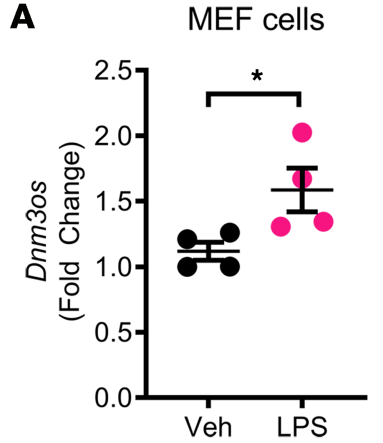

C

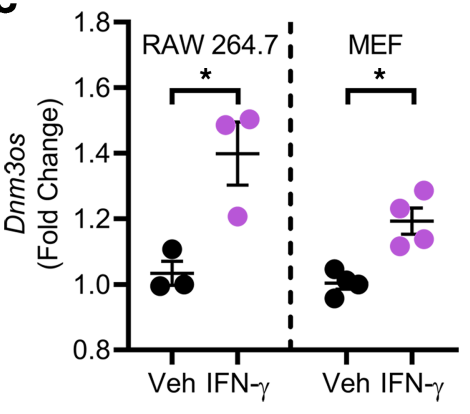

B

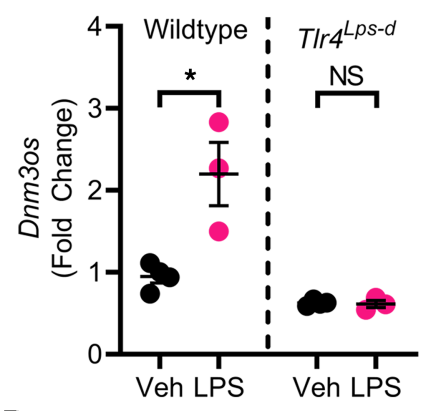

D

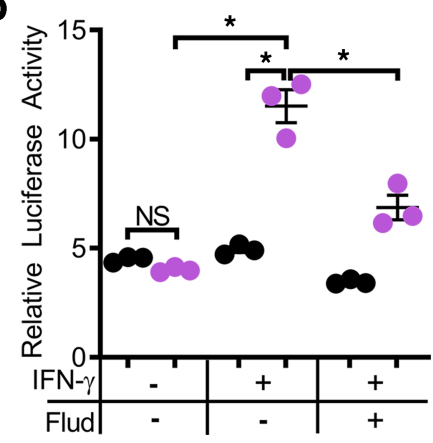

Figure 4. Proinflammatory TLR4 and IFN- $\gamma$ /STAT1 pathways transactivate the miR-214 host gene. (A) MEF cells were treated with LPS for 24 hours to activate TLR4. Q-PCR analysis showed increased Dnm3os expression in LPS-treated compared with vehicle-treated cells $(n=3)$. (B) Q-PCR analysis showed that Dnm3os expression was also increased in kidneys of WT mice $(n=3)$ injected with LPS. In contrast, LPS treatment did not cause Dnm3os upregulation in kidneys of TIr $4^{\text {Lps-d }}$ mice $(n=3)$, which harbor a spontaneous mutation in TIr 4 that makes them resistant to LPS. (C) MEF ( $n=$ 3) and RAW264.7 cells $(n=3)$ were treated with vehicle or IFN- $\gamma$. Compared with vehicle, IFN- $\gamma$ treatment increased Dnm3os expression in both MEF and RAW264.7 cells. (D) Dnm3os promoter contains a Stat1 binding site. Dnm3os promoter luciferase reporter assays were performed to determine whether the IFN- $\gamma /$ STAT1 pathway directly affects Dnm3os transcription. Relative luciferase activity of pGL3-Dnm3os (purple) and control pGL3-basic (black) was not different at baseline. Stimulation with IFN- $\gamma$ transactivated $\mathrm{pGI3-Dnm30s}$ but not the $\mathrm{pGL3}$-basic control. Treatment with Stat1 inhibitor, fludarabine (Flud), reversed the IFN- $\gamma$-mediated stimulation of pGL3-Dnm3os. ${ }^{*} P<0.05$; Student's unpaired $t$ test (A-C); 1-way ANOVA, Tukey's multiple-comparisons test (D); error bars indicate SEM.

IncRNA Dnm3os, which is also activated in ADPKD. A distinctive feature compared with the 2 other activated miRNAs, miR-17 92 and miR-21, that we have previously studied is miR-214's unique cellular source. Rather than cyst epithelial cells, stromal cells that surround the cysts produce this miRNA. Consistent with our findings, data from the GUDMAP show that Dnm3os expression is localized to mouse renal stromal cells $(29,30)$. A cellular atlas of the developing human kidney based on single-cell RNA-Seq also pointed to interstitial cells as the site of Dnm3os expression (31). Finally, miR-214 was identified as a kidney stroma-enriched miRNA based on the analysis of a mouse model that lacks the miRNA biogenesis enzyme DICER1 only in Foxd1-derived stromal cells (32). However, due to the drastic changes in signaling and cellular composition in the cystic kidneys, it is possible miR-214 is also produced by other cells in the cyst microenvironment. Indeed, Dnm3os and miR-214 expression is observed in macrophages and fibroblasts associated with cancer (33).

The second and somewhat unexpected finding of our work is that miR-214 is a protective and compensatory (albeit inadequate) signal emanating from the cyst microenvironment. Despite its upregulation, removing miR-214 from the cystic milieu worsened disease progression. This effect was observed irrespective of the type of gene mutation ( $P k d 1$ or $P k d 2$ ) or disease severity (aggressive or slowly progressing). To date, drug development has been geared toward targeting abnormal cyst-promoting pathways. Our work suggests an alternative approach that involves harnessing the therapeutic potential of the kidney's natural cyst-attenuating response. In this regard, an obvious but challenging question moving forward is whether drugs such as miRNA mimics designed to further enhance miR-214 activity are beneficial in ADPKD. Similar to our findings, miR-214 expression is increased in mouse models of acute kidney injury and fibrosis (34-36). However, miR-214 appears to produce divergent effects in kidney injury models. Whereas in mouse models, miR-214 deletion is beneficial, in a rat model of kidney injury, miR-214 inhibition is reported to be detrimental (35). 
A

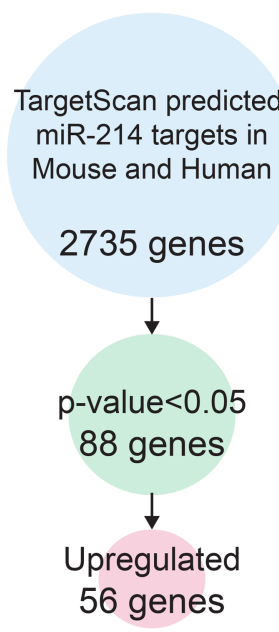

D

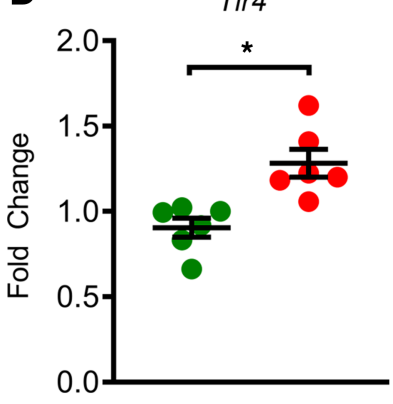

B

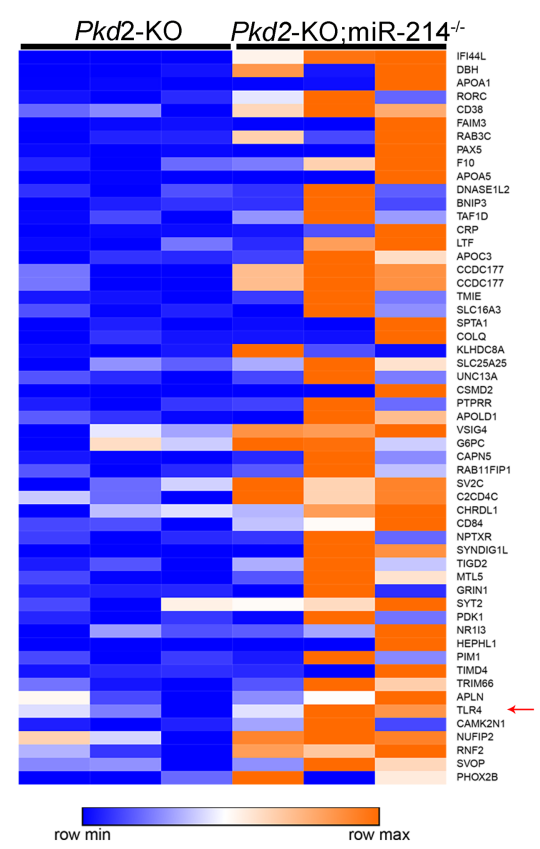

C

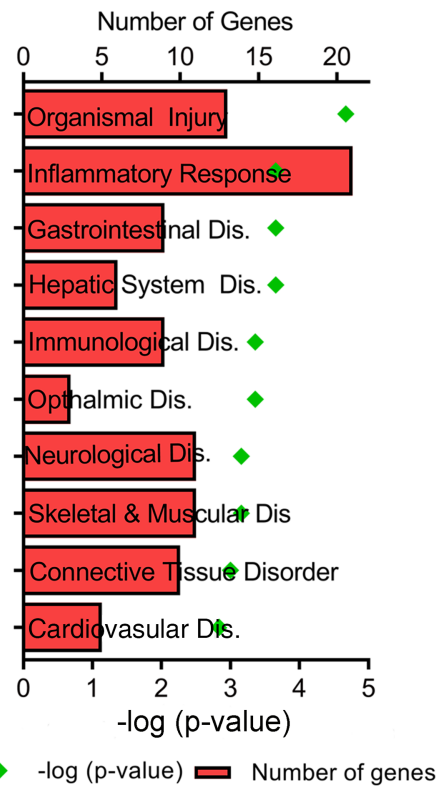

F

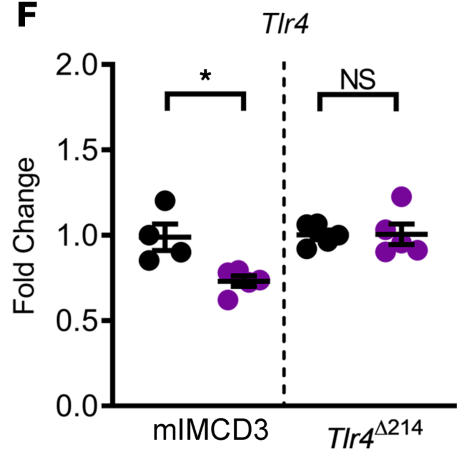

\section{$P k d 2-K O \quad O k d 2-K O ; m i R-214^{-1-}$}

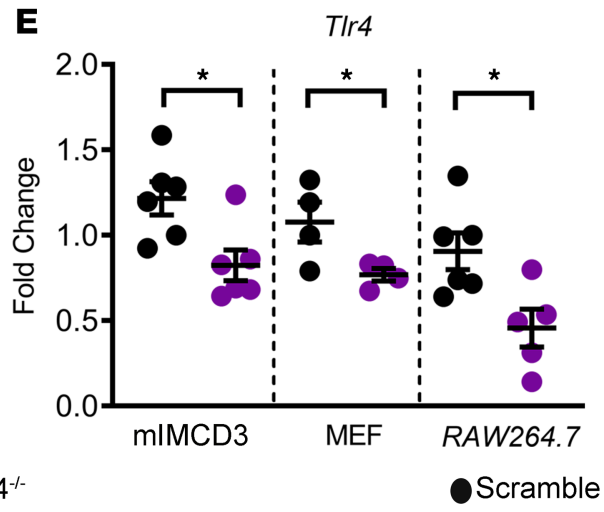

OmiR-214

Figure 5. miR-214 directly inhibits TLR4. (A) The approach used to identify direct miR-214 targets is shown. There were 2735 evolutionarily conserved miR-214 mRNA targets based on the prediction by TargetScan software. Intersecting this list with RNA-Seq data identified 88 mRNAs that were differentially expressed in Pkd2-KO miR-214 ${ }^{-/-}$kidneys compared with Pkd2-KO kidneys. Among these, 56 mRNAs were upregulated after miR-214 deletion in Pkd2-KO mice. (B) Heatmap showing upregulation of the 56 miR-214 targets in Pkd2-KO miR-214 ${ }^{-/-}(n=3)$ compared with Pkd2-KO kidneys $(n=3)$. Tlr4 was identified as one of the putative miR-214 targets (red arrow). (C) Analysis of the 56 upregulated mRNAs demonstrated that inflammation-associated pathways are the predominant signature of miR-214 deletion. (D) Q-PCR analysis confirmed that TIr4 expression was upregulated in Pkd2-KO miR-214-/- (red circles, $n=6$ ) compared with Pkd2-KO kidneys (green circles, $n=6$ ). (E) Q-PCR showing that, compared with scramble control, treatment with miR-214 mimic repressed TIr4 expression in mIMCD3 $(n=6), \operatorname{MEF}(n=4)$, and RAW 264.7 cells $(n=5)$. (F) To further validate TIr4 as a direct miR-214 target, we used CRISPR/Cas9 to generate a monoclonal mIMCD3 cell line with targeted deletion of the miR-214 binding site in T/r4 $3^{\prime}$ UTRs (T/r $\left.r^{\Delta \text { miR-214 }}\right)$. Q-PCR analysis showed that miR-214-mediated TIr4 repression was abolished in $T / r^{\Delta m i R-214}(n=5)$ but not in control mIMCD3 cells $(n=$ 4-5). ${ }^{*} P<0.05$; Student's unpaired $t$ test $(\mathbf{D}-\mathbf{F})$; error bars indicate SEM.

Finally, our work provides new insights into inflammation signaling in ADPKD. The canonical function of TLR4 signaling is in the innate immunity pathway against invading microbes (37). It is also activated by aseptic tissue damage/injury and aids in tissue regeneration (38-40). However, persistent TLR4 activation is often maladaptive. For example, TLR4 promotes kidney fibrosis in murine models of chronic kidney injury (41). Similarly, aberrant TLR4 activation in the tumor microenvironment and tumor-associated macrophages is thought to mediate anticancer immunity and cancer progression (42). We found that TLR4 activates miR-214 transcription. In turn, miR-214 directly inhibits TLR4. This miR-214-mediated negative feedback loop could function to dial down persistent TLR4 signaling and limit the maladaptive response and disease progression. Interestingly, thematically similar results are observed in a fish model of bacterial infection. Infection with Vibrio harveyi in miiuy croaker increases 
A

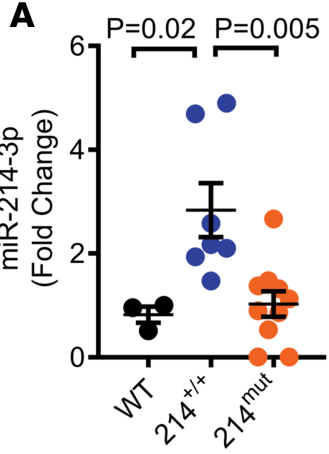

C

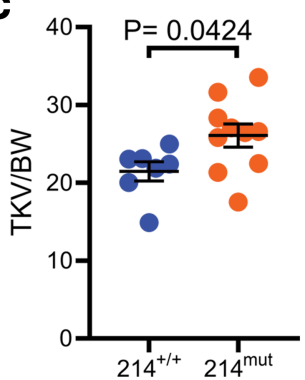

B

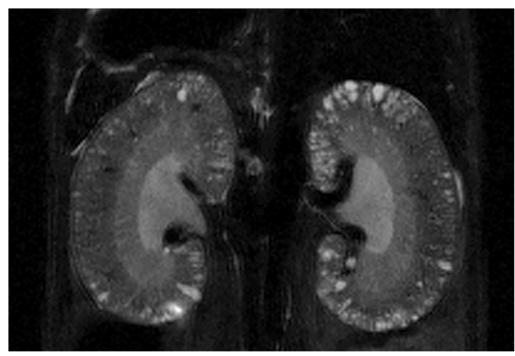

D

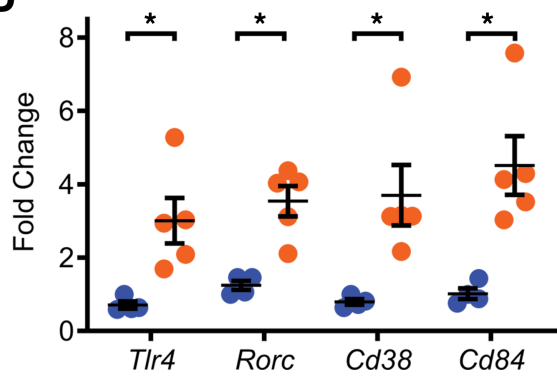

Pkd1 12C/Rc; miR-214 ${ }^{\text {mut }}$

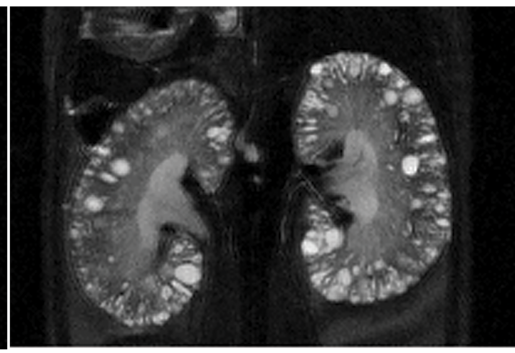

Pkd1 RC/RC; miR-214/++

Pkd1 RC/RC; miR-214

Figure 6. miR-214 deletion aggravates cyst growth in the Pkd1 $1^{R C / R C}$ model of ADPKD. The role of miR-214 was studied in a second ADPKD mouse model. miR-214 ${ }^{-1}$ mice were bred with $P k d 1^{R C / R C}$ mice to generate $P k d 7^{R C / R C}$ mice $(n=7)$ and $P k d 7^{R C / R C}$ miR-214 mut mice $(n=10)$ with deletion of either one $(n=$ 8) or both alleles $(n=2)$ of miR-214. (A) Q-PCR analysis showed that compared with kidneys from WT mice (black circles, $n=3$ ), miR-214 expression was increased in kidneys of 140-day-old $P k d 7^{R C / R C}$ mice (blue circles). miR-214 expression was reduced in kidneys of $P k d 7^{R C / R C}$ miR-214 ${ }^{\text {mut }}$ (orange circles) compared with $P k d 1^{R C / R C}$ mice (blue circles). (B) MRI was performed to determine total kidney volume of $P k d 1^{R C / R C}$ mice $(N=7)$ and $P k d 77^{R C / R C}$ miR-214 ${ }^{\mathrm{mut}}$ mice $(N=10)$ at 16 weeks of age. Representative MRI images corresponding to the mean of each group are shown. (C) Total kidney volume (TKV) normalized to BW was increased in $P k d 1^{R C / R C}$ miR-214 mut mice compared with $P k d 1^{R C / R C}$ mice. (D) Q-PCR analysis showing upregulation of miR-214 target genes, T/r4, Rorc, $C d 38$, and $C d 84$, in kidneys of 140 -day-old $P k d 1^{R C / R C}$ miR-214 ${ }^{\text {mut }}$ mice $(N=5)$ compared with $P k d 11^{R C / R C}$ mice $(N=4)$. ${ }^{*} P<0.05 ; 1$-way ANOVA, Tukey's multiple-comparisons test (A); Student's unpaired $t$ test (C and $\mathbf{D})$; error bars indicate SEM.

miR-214 expression (43). miR-214, in turn, restrains TLR signaling in this model by directly inhibiting Myd88, an essential TLR adapter protein that mediates downstream NF-kB signaling. Consistent with beneficial effects of miR-214 that we observed in ADPKD models, several studies have also reported tumor-suppressive effects of miR-214 in various cancers $(21,44)$.

Our study has raised several questions that remain unanswered. First, our work implicates stromal cells, but their role in ADPKD is unknown. Interestingly, a recent study found that removing interstitium enhanced the cyst growth of PKD organoids (10). It is, therefore, tempting to speculate that the stromal cells that produce miR-214 have a broadly protective function in ADPKD. Our study should prompt a closer examination of the role of stromal cells in tubular cyst growth. Second, while miR-214 deletion enhances cyst-associated $\mathrm{MRC1}^{+}$macrophages, it is unclear whether this is a cell-autonomous or a nonautonomous effect. It is wellknown that some miRNAs are released by cells that synthesize them and can be taken up by neighboring cells or even by cells at distant locations. Thus, miR-214 may be a paracrine signal that mediates the crosstalk between stromal, immune, and cyst epithelial cells to modulate the cyst microenvironment. Third, how TLR4 signaling affects ADPKD progression is unknown. The prediction would be that in an opposite effect to miR-214 deletion, loss of TLR4 will attenuate cyst growth. Fourth, as has been alluded to earlier, to validate miR-214 as a therapeutic target, it will be essential to test whether increasing miR-214 expression attenuates cyst growth. Finally, miRNAs generally regulate large gene networks. Therefore, miR-214 likely regulates many other mRNA targets in addition to Tlr4 in cystic kidneys. We identified several putative miR-214 targets from our unbiased transcriptomic analysis and validated 2 other immune-related, direct miR-214 mRNA targets. Nevertheless, the full scope of the biological pathways regulated by miR-214 in ADPKD is not known. Finally, miR-199a, which is cotranscribed with miR-214, and the host Dnm3os 1ncRNA itself, are implicated in inflammation $(20,45)$. To what extent they modulate the ADPKD phenotype is unknown.

In conclusion, we report a cyst-attenuating role of miR-214 in ADPKD. miR-214 is a stromal miRNA that is transactivated by proinflammatory signaling pathways. miR-214 functions to restrain 
cyst-associated inflammation by inhibiting TLR4. Thus, miR-214 is a protective molecular signal arising from the cyst microenvironment that attenuates cyst growth.

\section{Methods}

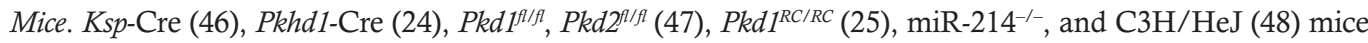
were used in this study. The generation of $P k h d 1$-Cre $P k d 2^{f / f l}$ miR-214 $4^{-/-}$mice and $P k d 1^{R C / R C}$ miR214 mut mice

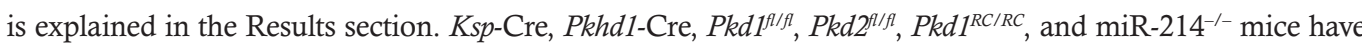
been maintained in a C57BL/6 background. Equal numbers of males and females were used in all studies. In the case of $P k d 1-\mathrm{KO}$ and $P k d 2-\mathrm{KO}$ mice, controls came from the same mouse line that lacks Cre recombinase. In the case of $P k d 1^{R C / R C}$ mice, negative controls were age-matched mice of the same background. To test whether TLR4 activation regulates miR-214 expression, we used C3H/HeJ mice, which have a spontaneous mutation in Tlr4 at the endotoxin (or LPS) response locus ( $T l r 4^{L p s-d}$ ) that makes these mice more resistant to endotoxin. C3H/HeJ or WT mice received an i.p. injection of $16 \mathrm{mg} / \mathrm{kg}$ ultrapure LPS from Escherichia coli O111:B4 (Ultrapure LPS-EB: tlrl-3pelps, InvivoGen) that activates only the TLR4, but not TLR2, pathway or vehicle and were killed after 24 hours. The ages at which mice were sacrificed are noted in the Results section and figures. All experiments involving animals were conducted under the approval of the UT Southwestern Institutional Animal Care and Use Committee.

Serum chemistries. BUN, aspartate aminotransferase, and alanine aminotransferase were measured using the Vitros 250 Analyzer.

Human specimens. Frozen ADPKD and NHK specimens were provided by the PKD Biomarkers and Biomaterials Core in the Kansas PKD Center at the Kansas University Medical Center (KUMC). Kidneys were obtained with the assistance of the Biospecimen Resource Core in the University of Kansas Cancer Center. In the operating room, the kidneys were immediately sealed in sterile bags, submerged in ice, and delivered to the laboratory. The protocol for the use of surgically discarded kidney tissues complied with federal regulations and was approved by the Institutional Review Board at KUMC.

Tissue harvesting and analysis. Mice were anesthetized using an approved protocol, and blood was obtained by cardiac puncture. The right kidney was flash frozen for further analysis, and the left kidney was perfused with cold $1 \times$ PBS and 4\% (wt/vol) paraformaldehyde. The left kidney and liver were then bisected; one section was sent for paraffin embedding, and the other was frozen using OCT. Sagittal sections of kidneys and liver were stained with $\mathrm{H} \& \mathrm{E}$ for analysis.

MRI. MRI and kidney volume calculations have been previously described (14). Briefly, MRI scans were performed using a 7T small-animal MRI scanner (Varian Inc., Agilent Technologies) equipped with a 40-mm Millipede RF coil (ExtendMR LLC). Animals received anesthesia ( $1 \%-3 \%$ isoflurane mixed in medical-grade oxygen) via inhalation through nose cone and were placed supine headfirst with the kidneys centered with respect to the center of the RF coil. MRI acquisitions were gated using respiratory triggering. The core temperature was kept at $28 \pm 1$ degrees centigrade. Two-dimensional scout images on the 3 orthogonal planes (axial, coronal, and sagittal) were acquired to ensure the positioning was correct. For kidney volume measurements, the high-resolution T2-weighted fast spin echo images were obtained on the coronal sections by applying a 6-ms sinc presaturation pulse to remove the fat signal.

$R N A$ isolation and Q-PCR. Total RNA was isolated from cultured cells or mouse kidneys using miRNeasy Mini kits (QIAGEN). First-strand cDNA was synthesized from mRNA using the Superscript First Strand cDNA synthesis kit (Invitrogen, Thermo Fisher Scientific), and Q-PCR was performed using the iQ SYBR Green Supermix (Bio-Rad). The Universal cDNA Synthesis kit from Exiqon was used for the first-strand synthesis from miRNA. The samples were loaded in triplicate on a CFX Connect Real-Time PCR Detection System (Bio-Rad). 18S and 5S RNA were used to normalize the expression of mRNA and miRNA, respectively. Data were analyzed using the Bio-Rad CFX software. The sequences of the PCR primers are shown in Supplemental Table 1.

$R N A$-Seq analysis. Strand-specific RNA-Seq libraries were prepared using the TruSeq Stranded Total RNA LT Sample Prep Kit from Illumina ( $n=3$ biological replicates for all groups). After quality check and quantification, libraries were sequenced at the UT Southwestern McDermott Center for Human Growth and Development using a Hiseq2500 Sequencer to generate 51-bp single-end reads. Before mapping, reads were trimmed to remove low-quality regions in the ends. Trimmed reads were mapped to the mouse genome (mm10) using TopHat version 2.0.12 with the UCSC iGenomes GTF file from Illumina. Alignments with mapping quality less than 10 were discarded. Expression abundance estimation and differential expression gene identification were 
done using edgeR. Genes with a $P$ value less than 0.05 were considered significantly differentially expressed between the 2 conditions. The identified genes were further subjected to Ingenuity Pathway Analysis (QIAGEN Inc.) (49). TargetScan was used to identify conserved miR-214 target genes.

Single-cell data analysis. The data set Combes et al. generated was obtained via the National Center for Biotechnology Information's (NCBI's) Gene Expression Omnibus under accession GSE108291 (26). The data were processed using the scran Bioconductor package (50). The data consisted of unfiltered feature-barcode matrices generated by running the CellRanger count pipeline. Cells were called from empty droplets by testing for the deviation of the expression profile for each cell from the ambient RNA pool (51). Cells with large mitochondrial proportions, i.e., more than 3 mean absolute deviations away from the median, were removed. Cells were preclustered, a deconvolution method was applied to compute size factors for all cells (52), and normalized log expression values were calculated. Variance was partitioned into technical and biological components by assuming technical noise was Poisson distributed and attributing any estimated variance in excess of that accounted for by a fitted Poisson trend to biological variation. The dimensionality of the data set was reduced by performing principal component analysis and discarding the latter principal components for which the variance explained was less than variance attributable to technical noise. Masking of biological effects by expression changes due to the cell cycle phase was mitigated by blocking on this covariate. The cell cycle phase was inferred using the pair-based classifier implemented in the cyclone function of scran. Corrected log-normalized expression counts were obtained by calling the removeBatchEffect from the limma (53) Bioconductor package with a design formula including $\mathrm{G}_{1}$ and $\mathrm{G}_{2} \mathrm{M}$ cell cycle phase scores as covariates. Cells were clustered by building a shared nearest neighbor graph (54) and executing the Walktrap algorithm (55). We performed partition-based graph abstraction to model the connectivity between clusters (56). At cluster resolution, the edge score threshold was varied until the graph was decomposed into connected components. Evaluation of marker gene expression within the components permitted categorizing as epithelium, leukocyte, erythrocyte, endothelium, and stroma. Differential gene expression analysis was performed using the 2-part generalized linear model that concurrently models expression rate above background and expression mean implemented in MAST (57). A one-versus-all strategy was employed comparing each component to all other components.

In situ hybridization. We subcloned 918 bp of Dnm3os into the pXY vector using the following primers: Dnm3os forward, TAGAATGTCGACTATGGTAAATTGTGATTTTCTGTGTGT, and Dnm3os reverse, TAAGATGCGGCCGCACATTTTCTCCCAGAACTTTCTTTAAC, where the underlined segments represent the NotI and Sall restriction enzyme sites. In situ hybridization was performed as previously described (58).

Immunofluorescence staining. The anti-MRC1 antibody (Abcam 64693; 1:100 dilution) was used on paraffin-embedded sections.

Western blot analysis. Total protein was extracted from mouse kidneys. Between 10 and $20 \mu \mathrm{g}$ of protein was loaded onto a $4 \%-15 \%$ polyacrylamide gel. The protein was transferred to a nitrocellulose membrane. The membrane was blocked with $5 \%$ bovine serum albumin and probed overnight at $4^{\circ} \mathrm{C}$ with anti-MRC1 (Abcam 64693; 1:1000), anti-phospho-Stat1 (Abcam 29045; 1:1000), and anti-actin antibodies (MilliporeSigma a3854; 1:40,000). Goat anti-rabbit HRP-conjugated IgG was used as a secondary antibody (Invitrogen, Thermo Fisher Scientific, G21234), and the blots were developed using SuperSignal West Dura Extended Dilution substrate or enhanced chemiluminescence. The protein bands were quantified using Quantity One imaging software from Bio-Rad.

Cell culture. mIMCD3, RAW264.7, and MEFs were obtained from ATCC and grown in DMEM low-glucose medium supplemented with $10 \% \mathrm{FBS}$ and maintained in $5 \% \mathrm{CO}_{2}$ atmosphere at $37^{\circ} \mathrm{C}$. RAW264.7 cells were treated with IL-4 (MilliporeSigma) or IFN- $\gamma$ (Cell Signaling Technology) or $10 \mathrm{mM}$ miR-214 mimic (Dharmacon) for 48 hours.

Plasmids. Dnm3os promoter was amplified using the forward and reverse primers that introduced Mlu1 and Xho1 restriction sites at the $5^{\prime}$ and $3^{\prime}$ ends, respectively. The sequences of the forward and the reverse primers were TAGAATACGCGTCAGTGTGGGTGCTTGAAGTC and TAGAATCTCGAGATTTCCAGTCTTGACGTGGC, where the underlined sequence is the Mlu1 and Xho1 site, respectively. The PCR product was ligated into the pGL3-basic plasmid to generate a pGL-Dnm3os plasmid.

Luciferase assay. MEF cells were plated in 6-well dishes $\left(2 \times 10^{5}\right.$ cells per well $)$ and cotransfected with $0.6 \mu \mathrm{g}$ pGL3 plasmids encoding Photinus luciferase and $0.02 \mu \mathrm{g}$ pR-CMV vector encoding Renilla. 
After 6 hours, cell medium was replaced with fresh medium containing $0.66 \mu \mathrm{g} / \mathrm{mL}$ of IFN- $\gamma$ (Cell Signaling Technology) and/or $20 \mu \mathrm{M}$ fludarabine (MilliporeSigma). Forty-eight hours later, the cells were lysed in $250 \mu \mathrm{L}$ of passive lysis buffer, and $40 \mu \mathrm{L}$ of the cell lysate was added to 96-well plates. Photinus and Renilla luciferase activities were measured by using the Dual-Luciferase Reporter Assay System (Promega Corp.) according to the manufacturer's directions. All experiments were performed at least 3 times in triplicate.

Tlr4 3' UTR studies. sgRNAs were designed to induce Cas9-mediated DNA cleavage within the miR214 binding site in the Tlr4 3' UTR. sgRNAs were subcloned into pX330mCherry as previously described (59). pX330mCherry plasmid containing an sgRNA specific to the Tlr4 miR-214 binding site was transfected into mIMCD3 cells. After 48 hours, mCherry-positive cells were FACS sorted and plated individually in a 96-well plate. Colonies were screened for mutation of the Tlr4 miR-214 binding site using PCR and subsequent sequencing. We used a cell line in which miR-214 binding was deleted as confirmed by sequencing.

Data availability. RNA-Seq data have been deposited in the NCBI's Gene Expression Omnibus repository under accession number GSE137945.

Statistics. Data shown are the mean with error bars indicating the SEM. Statistical analysis was performed using 2-tailed Student's $t$ test for pairwise comparison or 1-way ANOVA followed by Tukey's post hoc test for multiple comparisons. Survival analysis was performed using the Mantel-Cox test. $P<0.05$ was considered statistically significant.

Study approval. All experiments involving animals were conducted under the approval of the UT Southwestern Institutional Animal Care and Use Committee. The protocol for the use of surgically discarded kidney tissues complied with the federal regulations and was approved the Institutional Review Board at the University of Kansas Medical Center.

\section{Author contributions}

RL, MY, and VP designed research. RL, MY, AF, HR, KA, SF, CC, AM, and LB performed experiments. RL, MY, AF, HR, KA, SF, CC, TC, PI, and VP interpreted the data. DPW and PI provided critical reagents. RL and MY prepared the figures, and RL and VP wrote the manuscript. All authors contributed critical edits to the paper.

\section{Acknowledgments}

The work is supported by the National Institutes of Health (R01DK102572) and the Department of Defense (D01 W81XWH1810673) to VP. RL is supported by National Institutes of Health (K08DK117049). SF was supported by the Charles and Jane Pak Center for Mineral Metabolism and Clinical Research Innovative Research Support Award. We thank the UT Southwestern O'Brien Kidney Research Core Center (P30DK079328) and UT Southwestern Bioinformatics Core Facility for providing critical reagents and services. We thank the PKD Biomarkers and Biomaterials Core of the University of Kansas Medical Center (P30DK106912) and the Mayo Translational PKD center for providing the human ADPKD samples.

Address correspondence to: Vishal Patel, Department of Internal Medicine and Division of Nephrology, University of Texas Southwestern Medical Center, 5323 Harry Hines Blvd., Dallas, Texas 75390, USA. Phone: 214.648.0369; Email: vishald.patel@utsouthwestern.edu.

1. Torres VE, Harris PC, Pirson Y. Autosomal dominant polycystic kidney disease. Lancet. 2007;369(9569):1287-1301.

2. Patel V, Chowdhury R, Igarashi P. Advances in the pathogenesis and treatment of polycystic kidney disease. Curr Opin Nephrol Hypertens. 2009;18(2):99-106.

3. Harris PC, Torres VE. Genetic mechanisms and signaling pathways in autosomal dominant polycystic kidney disease. J Clin Invest. 2014;124(6):2315-2324

4. Swenson-Fields KI, et al. Macrophages promote polycystic kidney disease progression. Kidney Int. 2013;83(5):855-864.

5. Chen L, et al. Macrophage migration inhibitory factor promotes cyst growth in polycystic kidney disease. J Clin Invest. 2015;125(6):2399-2412.

6. Karihaloo A, et al. Macrophages promote cyst growth in polycystic kidney disease. J Am Soc Nephrol. 2011;22(10):1809-1814.

7. Zimmerman KA, et al. Single-cell RNA sequencing identifies candidate renal resident macrophage gene expression signatures across species. J Am Soc Nephrol. 2019;30(5):767-781.

8. Yang Y, et al. Interactions between macrophages and cyst-lining epithelial cells promote kidney cyst growth in Pkd1-deficient mice. J Am Soc Nephrol. 2018;29(9):2310-2325.

9. Kleczko EK, et al. CD8 ${ }^{+} \mathrm{T}$ cells modulate autosomal dominant polycystic kidney disease progression. Kidney Int. 2018;94(6):1127-1140.

10. Cruz NM, et al. Organoid cystogenesis reveals a critical role of microenvironment in human polycystic kidney disease. 
Nat Mater. 2017;16(11):1112-1119.

11. Bartel DP. MicroRNAs: target recognition and regulatory functions. Cell. 2009;136(2):215-233.

12. Baek D, Villén J, Shin C, Camargo FD, Gygi SP, Bartel DP. The impact of microRNAs on protein output. Nature. 2008;455(7209):64-71.

13. Yheskel M, Patel V. Therapeutic microRNAs in polycystic kidney disease. Curr Opin Nephrol Hypertens. 2017;26(4):282-289.

14. Hajarnis S, et al. microRNA-17 family promotes polycystic kidney disease progression through modulation of mitochondrial metabolism. Nat Commun. 2017;8:14395.

15. Lakhia R, et al. MicroRNA-21 aggravates cyst growth in a model of polycystic kidney disease. J Am Soc Nephrol. 2016;27(8):2319-2330.

16. Kim DY, et al. Impact of miR-192 and miR-194 on cyst enlargement through EMT in autosomal dominant polycystic kidney disease. FASEB J. 2019;33(2):2870-2884

17. Lee EC, et al. Discovery and preclinical evaluation of anti-miR-17 oligonucleotide RGLS4326 for the treatment of polycystic kidney disease. Nat Commun. 2019;10(1):4148.

18. Loebel DA, Tsoi B, Wong N, Tam PP. A conserved noncoding intronic transcript at the mouse Dnm3 locus. Genomics. 2005;85(6):782-789.

19. Aboudehen K, et al. Long noncoding RNA Hoxb3os is dysregulated in autosomal dominant polycystic kidney disease and regulates mTOR signaling. J Biol Chem. 2018;293(24):9388-9398.

20. Das S, et al. Diabetes mellitus-induced long noncoding RNA Dnm3os regulates macrophage functions and inflammation via nuclear mechanisms. Arterioscler Thromb Vasc Biol. 2018;38(8):1806-1820.

21. Wu D, Lu P, Mi X, Miao J. Exosomal miR-214 from endometrial stromal cells inhibits endometriosis fibrosis. Mol Hum Reprod. 2018;24(7):357-365

22. Wang P, et al. miR-214/199a/199a* cluster levels predict poor survival in hepatocellular carcinoma through interference with cell-cycle regulators. Oncotarget. 2016;7(1):929-945.

23. Xia H, Ooi LL, Hui KM. MiR-214 targets $\beta$-catenin pathway to suppress invasion, stem-like traits and recurrence of human hepatocellular carcinoma. PLoS One. 2012;7(9):e44206.

24. Williams SS, et al. Tissue-specific regulation of the mouse Pkhd1 (ARPKD) gene promoter. Am J Physiol Renal Physiol. 2014;307(3):F356-F368.

25. Hopp K, et al. Functional polycystin-1 dosage governs autosomal dominant polycystic kidney disease severity. J Clin Invest. 2012;122(11):4257-4273.

26. Combes AN, et al. Single cell analysis of the developing mouse kidney provides deeper insight into marker gene expression and ligand-receptor crosstalk. Development. 2019;146(12):dev178673.

27. Strauss L, et al. RORC1 regulates tumor-promoting "emergency" granulo-monocytopoiesis. Cancer Cell. 2015;28(2):253-269.

28. Chini EN, Chini CCS, Espindola Netto JM, de Oliveira GC, van Schooten W. The pharmacology of CD38/NADase: an emerging target in cancer and diseases of aging. Trends Pharmacol Sci. 2018;39(4):424-436.

29. Harding SD, et al. The GUDMAP database--an online resource for genitourinary research. Development. 2011;138(13):2845-2853

30. McMahon AP, et al. GUDMAP: the genitourinary developmental molecular anatomy project. J Am Soc Nephrol. 2008;19(4):667-671.

31. Brunskill EW, et al. Atlas of gene expression in the developing kidney at microanatomic resolution. Dev Cell. 2008;15(5):781-791.

32. Nakagawa $\mathrm{N}$, et al. Dicer1 activity in the stromal compartment regulates nephron differentiation and vascular patterning during mammalian kidney organogenesis. Kidney Int. 2015;87(6):1125-1140.

33. Zhang $\mathrm{H}$, et al. Cancer-associated fibroblast-promoted LncRNA DNM3OS confers radioresistance by regulating DNA damage response in esophageal squamous cell carcinoma. Clin Cancer Res. 2019;25(6):1989-2000.

34. Denby L, et al. MicroRNA-214 antagonism protects against renal fibrosis. J Am Soc Nephrol. 2014;25(1):65-80.

35. Zhu X, Li W, Li H. miR-214 ameliorates acute kidney injury via targeting DKK3 and activating of Wnt/ $\beta$-catenin signaling pathway. Biol Res. 2018;51(1):31.

36. Bai M, et al. MicroRNA-214 promotes chronic kidney disease by disrupting mitochondrial oxidative phosphorylation. Kidney Int. 2019;95(6):1389-1404

37. Kawai T, Akira S. Toll-like receptors and their crosstalk with other innate receptors in infection and immunity. Immunity. 2011;34(5):637-650

38. Wang D, et al. Toll-like receptor 4 mediates the regenerative effects of bone grafts for calvarial bone repair. Tissue Eng Part A 2015;21(7-8):1299-1308.

39. Kulkarni OP, et al. Toll-like receptor 4-induced IL-22 accelerates kidney regeneration. J Am Soc Nephrol. 2014;25(5):978-989.

40. Molteni M, Gemma S, Rossetti C. The role of Toll-like receptor 4 in infectious and noninfectious inflammation. Mediators Inflamm. 2016;2016:6978936.

41. Ma J, et al. TLR4 activation promotes podocyte injury and interstitial fibrosis in diabetic nephropathy. PLoS One. 2014;9(5):e97985.

42. Murad S. Toll-like receptor 4 in inflammation and angiogenesis: a double-edged sword. Front Immunol. $2014 ; 5: 313$.

43. Chu Q, Sun Y, Cui J, Xu T. Inducible microRNA-214 contributes to the suppression of NF-kB-mediated inflammatory response via targeting myd88 gene in fish. J Biol Chem. 2017;292(13):5282-5290.

44. Yang Y, Liu Y, Li G, Li L, Geng P, Song H. microRNA-214 suppresses the growth of cervical cancer cells by targeting EZH2. Oncol Lett. 2018;16(5):5679-5686.

45. Bardin P, et al. Small RNA and transcriptome sequencing reveal the role of miR-199a-3p in inflammatory processes in cystic fibrosis airways. J Pathol. 2018;245(4):410-420

46. Shao X, Somlo S, Igarashi P. Epithelial-specific Cre/lox recombination in the developing kidney and genitourinary tract. $J$ Am Soc Nephrol. 2002;13(7):1837-1846.

47. Wu G, et al. Somatic inactivation of $\mathrm{Pkd} 2$ results in polycystic kidney disease. Cell. 1998;93(2):177-188.

48. Akeson EC, et al. Chromosomal inversion discovered in C3H/HeJ mice. Genomics. 2006;87(2):311-313

49. Krämer A, Green G, Pollard J Jr, Tugendreich S. Causal analysis approaches in Ingenuity Pathway Analysis. Bioinformatics. 
2014;30(4):523-530.

50. Lun AT, McCarthy DJ, Marioni JC. A step-by-step workflow for low-level analysis of single-cell RNA-seq data with Bioconductor. F1000Res. 2016;5:2122.

51. Lun ATL, et al. EmptyDrops: distinguishing cells from empty droplets in droplet-based single-cell RNA sequencing data. Genome Biol. 2019;20(1):63.

52. Lun AT, Bach K, Marioni JC. Pooling across cells to normalize single-cell RNA sequencing data with many zero counts Genome Biol. 2016;17:75.

53. Ritchie ME, et al. limma powers differential expression analyses for RNA-sequencing and microarray studies. Nucleic Acids Res. 2015;43(7):e47.

54. Xu C, Su Z. Identification of cell types from single-cell transcriptomes using a novel clustering method. Bioinformatics. 2015;31(12):1974-1980

55. Pons P, Latapy M. Computing communities in large networks using random walks. J Graph Algorithms Appl. 2006;10(2):191-218.

56. Wolf FA, et al. PAGA: graph abstraction reconciles clustering with trajectory inference through a topology preserving map of single cells. Genome Biol. 2019;20(1):59.

57. Finak G, et al. MAST: a flexible statistical framework for assessing transcriptional changes and characterizing heterogeneity in single-cell RNA sequencing data. Genome Biol. 2015;16:278

58. Das A, et al. Stromal-epithelial crosstalk regulates kidney progenitor cell differentiation. Nat Cell Biol. 2013;15(9):1035-1044

59. Zhang F, Wen Y, Guo X. CRISPR/Cas9 for genome editing: progress, implications and challenges. Hum Mol Genet. 2014;23(R1):R40-R46. 